Published in final edited form as:

J Am Geriatr Soc. 2011 July ; 59(7): 1326-1331. doi:10.1111/j.1532-5415.2011.03480.x.

\title{
Timing of Do-Not-Resuscitate Orders for Hospitalized Older Adults Who Require a Surrogate Decision-Maker
}

\author{
Alexia M. Torke, MD, MS ${ }^{*},, \dagger$, Greg A. Sachs, MD ${ }^{*}, \neq$, Paul R. Helft, MD $\S, \dagger$, Sandra Petronio,

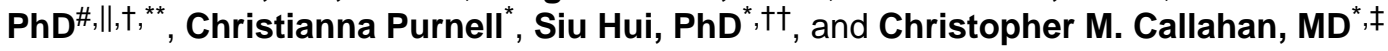 \\ "Center for Aging Research, Regenstrief Institute, Indiana University, Indianapolis, Indiana \\ ¥Division of General Internal Medicine and Geriatrics, Indiana University, Indianapolis, Indiana \\ †'Division of Biostatistics, School of Medicine, Indiana University, Indianapolis, Indiana \\ §Melvin and Bren Simon Cancer Center, Indiana University, Indianapolis, Indiana \\ IISchool of Nursing, Indiana University, Indianapolis, Indiana \\ \#School of Informatics, Indiana University, Indianapolis, Indiana \\ †Fairbanks Center for Medical Ethics, IU Health, Indianapolis, Indiana \\ **Department of Communication Studies, Indiana University-Purdue University Indianapolis, \\ Indiana
}

\begin{abstract}
OBJECTIVES-To examine the frequency of surrogate decisions for in-hospital do-notresuscitate (DNR) orders and the timing of DNR order entry for surrogate decisions.
\end{abstract}

DESIGN-Retrospective cohort study.

SETTING-Large, urban, public hospital.

PARTICIPANTS-Hospitalized adults aged 65 and older over a 3-year period (1/1/200412/31/2006) with a DNR order during their hospital stay.

\footnotetext{
(C) 2011, Copyright the Authors

Address correspondence to Alexia Torke, IU Center for Aging Research, 410 W, 10th St., Indianapolis, IN 46202. atorke@iupui.edu. This research was presented in part at the National Meeting of the Society of General Internal Medicine, Minneapolis, Minnesota, April 27 to May 1, 2010.

Author Contributions: Study concept and design: Torke, Sachs, Callahan. Acquisition of subjects and/or data: Torke, Purnell. Analysis and interpretation of data: Torke, Sachs, Helft, Petronio, Purnell, Hui, Callahan. Preparation of manuscript: Torke, Sachs, Helft, Petronio, Purnell, Hui, Callahan.

Conflict of Interest: This research was supported by Award P30AG024967 from the National Institute on Aging (NIA) to the Indiana University Roybal Center. The content is solely the responsibility of the authors and does not necessarily represent the official views of the NIA or the National Institutes of Health. Dr. Torke was supported by a Geriatrics Health Outcomes Research Scholars Award sponsored by the American Geriatrics Society and the John A. Hartford Foundation and currently holds a career development award from the NIA (K23AG031323). Dr. Callahan is supported by a Midcareer Investigator Award in Patient-Oriented Research (K24 4682309).

Dr. Sachs serves as a consultant to CVS Caremark's National Pharmacy and Therapeutics Committee. This involves the review of medications for inclusion on national formulary and other medication lists with criteria on safety and efficacy (strict firewall exists between work of the committee and business operations, contracts, and relationships between CVS Caremark, clients, and pharmaceutical firms). He receives an honorarium that in some years may exceed $\$ 10,000$ if he attends all meetings and performs additional telephone consultations. He has received grants within the last 3 years from the Alzheimer's Association, National Institutes of Health, Agency for Healthcare Research and Quality, National Palliative Care Research Center, Donald W. Reynolds Foundation, and John A. Hartford Foundation.
} 
MEASUREMENTS-Electronic chart review provided data on frequency of surrogate decisions, patient demographic and clinical characteristics, and timing of DNR orders.

RESULTS-Of 668 patients, the ordering physician indicated that the DNR decision was made with the patient in 191 cases (28.9\%), the surrogate in 389 (58.2\%), and both in 88 (13.2\%). Patients who required a surrogate were more likely to be in the intensive care unit $(62.2 \%$ vs $39.8 \%, P<.001)$ but did not differ according to demographic characteristics. By hospital Day 3 , $77.6 \%$ of patient decisions, $61.9 \%$ of surrogate decisions, and $58.0 \%$ of shared decisions had been made. In multivariable models, the number of days from admission to DNR order was higher for surrogate (odds ratio $(\mathrm{OR})=1.97, P<.001)$ and shared decisions $(\mathrm{OR}=1.48, P=.009)$ than for patient decisions. The adjusted hazard ratio for hospital death was higher for patients with surrogate than patient decisions $(2.61,95 \%$ confidence interval $(\mathrm{CI})=1.56-4.36)$. Patients whose DNR orders were written on Day 6 or later were twice as likely to die in the hospital $(\mathrm{OR}=2.20$, 95\% CI $=1.45-3.36$ ) than patients with earlier DNR orders.

CONCLUSION-For patients who have a DNR order entered during their hospital stay, order entry occurs later when a surrogate is involved. Surrogate decision-making may take longer because of the greater ethical, emotional, or communication complexity of making decisions with surrogates than with patients.

\section{Keywords}

resuscitation; decision-making; proxy; end of life

When an older adult is admitted to the hospital, it is important to consider whether cardiopulmonary resuscitation (CPR) is appropriate in the event of cardiac or respiratory arrest. It is generally accepted that patients or their surrogate decision-makers should be included in such decisions, to give the patient or family the opportunity to refuse an intervention that may be unwanted or medically inappropriate, but there is evidence that communication and decision-making about code status is often of poor quality. Several studies have found low levels of chart documentation of code status discussions with hospitalized patients within 24 hours of admission, ${ }^{1}$ with patients who died, ${ }^{2}$ and with patients who had undergone resuscitation. ${ }^{3}$ This suggests that communication about code status did not occur in many cases or was not documented so as to guide future patient care. A study that audio-recorded code status discussions by medical residents found that most left out important information about CPR and did not address likely patient outcomes. ${ }^{4}$ There is also evidence that many do-not-resuscitate (DNR) orders are written close to death. ${ }^{2,5-9}$ Although there are many possible reasons for this, including sudden or unexpected deterioration in clinical status and the sensitive nature of these discussions, ${ }^{2}$ failure to address end-of-life issues in a timely manner may also contribute. ${ }^{7}$

Although making DNR decisions is always complex, there is evidence that the communication and decision-making process is even more difficult when patients are unable to participate and physicians must discuss the decision with a surrogate. ${ }^{10,11}$ In the intensive care unit (ICU), where surrogate decision-making is common, up to one-third of families report problems with communication and decision-making. ${ }^{12}$ Physicians engaged in surrogate decision-making also report that communication with surrogates is often delayed or ineffective. ${ }^{13}$ Family members who are called upon to make end-of-life decisions report high levels of distress ${ }^{14}$ and describe strong emotions such as guilt and ambivalence when asked to make decisions about code status. ${ }^{15}$ These additional complexities of surrogate decision-making are important because as many as $40 \%$ of hospitalized adults are unable to make their own medical decisions, ${ }^{16}$ and studies of DNR decision-making have found that families make the decision from one-third to more than three-quarters of the time. , $^{5,17}$ 
Surrogate decision-making is likely to become even more common as the population ages and the prevalence of dementia and delirium increases.

In spite of the frequency and added complexity of surrogate decision-making, there are few data on the characteristics and outcomes of older adults who require a surrogate decisionmaker for code status decisions or on whether having a surrogate is associated with differences in the process of writing a DNR order. To address these issues, a retrospective cohort study of adults 65 and older was conducted to examine the frequency of surrogate decisions for in-hospital DNR orders, to compare the characteristics of patients who require surrogate decisions with those of patients who do not, and to determine whether timing of the DNR order entry differs when surrogates are involved. It was hypothesized that DNR orders would be written later in the hospital course and closer to death for patients who required a surrogate than for those who did not.

\section{METHODS}

\section{Study Design and Setting}

A retrospective chart review of electronic medical records (EMR) was conducted for all inpatients 65 and older admitted to Wishard Hospital from January 1, 2004, to December 31, 2006. WMH is a 450-bed, university-affiliated, urban, public hospital. WMH is closely affiliated with the Regenstrief Institute, Inc., which administers the EMR and Computerized Physician Order Entry (CPOE) Systems. The EMR captures hospital data about patient demographics, dates of admission and discharge, hospital costs, and hospital deaths. This information is merged at the patient level with mortality data from the Indiana State Board of Health. The EMR also captures all orders for each patient entered through the hospital CPOE system, including all DNR orders. No paper orders are entered for hospitalized patients, so data capture for DNR orders is complete.

\section{Subject Selection}

Using the EMR, all patients who had a DNR order written during their hospital stay were identified. For patients with multiple admissions, the first admission in the study period during which a DNR order was written was selected. Patients were excluded if information regarding whether a surrogate was involved in the discussion of the DNR order was missing or if they were a prisoner during the hospital stay.

\section{Data Collection}

The primary independent variable addressed whether the ordering physician discussed the DNR order with the patient or a surrogate. This was determined from a text field in the DNR order that asked the entering physician to indicate whether she or he discussed the order with the patient alone (patient decisions), with a surrogate decision-maker alone (surrogate decisions), or with both the patient and a surrogate decision-maker (shared decisions). Data on the hospital day on which the DNR order was written were collected, and the number of days from admission to first DNR order was determined. The EMR also provide data on days of admission and discharge and in-hospital mortality, which allowed hospital length of stay and time from DNR order to discharge or death to be calculated. Patient demographic information, including age, race, and receipt of Medicaid insurance (as a proxy for low socioeconomic status (SES)) were also collected.

\section{Statistical Analysis}

Descriptive statistics were calculated for patient characteristics and clinical outcomes in the three groups based on DNR decision-making (by patient, by surrogate, or shared). The 
descriptive measures were compared between groups using Fisher exact tests for proportions and Wilcoxon tests for continuous variables.

To examine the length of time taken to reach DNR decisions, the distribution of hospital day on which the first DNR order occurred was plotted for the groups according to patient decision, surrogate decision, and shared decision. These were compared using a Wilcoxon test. Negative binomial models were then used to examine the difference in the number of days from admission to first DNR order, controlling for demographic variables including age, race, and SES (using Medicaid insurance as a proxy for low SES) and any ICU stay during the target hospitalization. Negative binomial models are appropriate when the outcome is positively skewed and follows a Poisson distribution with overdispersion (variance>mean). ${ }^{18}$ Survival analysis approaches were not used because group assignment (patient, surrogate, or shared) was determined retrospectively at the time of the event (DNR order) rather than prospectively. Additionally, the cohort included only patients whose events were uncensored (i.e., a DNR order was written for all subjects) rather than all subjects who were at risk for the event. Goodness of fit was assessed according to the deviance of the model. When analyses were repeated with outliers removed (246 and 366 days to DNR), results were similar and are not reported in the manuscript. To investigate the potential relationships between the decision-maker and timing of DNR orders and the patient's time to death, survival analysis was used to compare the time from admission to inhospital death and time from first DNR order to death of the three decision groups. Hospital discharge was censored in both analyses. Differences in the Kaplan-Meier curves were tested using log rank tests, followed by Cox proportional hazard models to control for age, race, sex, and SES. The variable "hospital day of DNR order" was then dichotomized into two groups: less than 6 days (early DNR orders; $77.4 \%$ of subjects) and 6 days or longer (late DNR orders, 22.6\%). Logistic regression was conducted to determine the odds of hospital death based on late DNR decisions, controlling for demographic variables. All data analysis was conducted using SAS 9.2 (SAS Institute, Inc., Cary, NC).

\section{RESULTS}

\section{Surrogate Characteristics}

Of 6,143 admissions over the 3-year period, there were 956 DNR orders for 725 unique patients. The first DNR order was selected for patients with more than one order. Of the 725 patients, 57 had missing data or were ineligible, leaving a final sample of 668 patients. Subjects were $64 \%$ female, $45 \%$ white, and 50\% African American (Table 1). Mortality was $24 \%$ during hospitalization, and $45 \%$ of patients died by 30 days after admission.

\section{Frequency of DNR Decision-Making}

The ordering physician indicated that the DNR decision was discussed with patients in 191 cases (28.2\%), surrogates in 389 (58.2\%), and patients and surrogates in 88 cases (13.2\%) (Table 1). Patients who required a surrogate decision were more likely to be in the ICU at some point during hospitalization $(62.2 \%$ vs $39.8 \%, P<.001)$ but did not differ according to age, sex, race, or Medicaid status. Patients with shared decisions did not differ in terms of demographic variables, length of stay, or likelihood of ICU stay from those who made their own decisions.

\section{Timing of DNR Decision-Making and Death}

The mean hospital day of DNR order was significantly later for surrogate decisions (6.6 days) than for patient decisions ( 3.2 days) $(P=.002)$ and for shared decisions (4.4 days) than patient decisions (3.2 days) $(P=.002)$. The distributions of the hospital day of DNR order in the three groups show that the proportion of DNR orders made in the first 3 hospital days 
was highest in the patient decision group, whereas the other two groups had higher percentages of DNR orders written on or after Day 4 (Figure 1). By hospital Day 3, 77.6\% of patient DNR decisions, $61.9 \%$ of surrogate decisions, and $58.0 \%$ of shared decisions had been made. By Day 6, 90.5\% of patient decisions, $77.6 \%$ of surrogate decisions, and $76.2 \%$ of shared decisions had been made. Using a negative binomial model to control for demographic variables, it was found that differences in the time from admission to DNR orders between surrogate and patient decisions remained significant (odds ratio $(\mathrm{OR})=1.97$, $P<.001)$ and between shared and patient decisions $(\mathrm{OR}=1.48, P=.009)$. In addition, the time from admission to DNR order was shorter for women than men $(\mathrm{OR}=0.67, P<.001)$ but was longer for patients with an ICU stay than for those on the medical wards (OR $=$ $1.40, P<.001)$. There were no differences according to age, race, or Medicaid status.

Patients who required a surrogate had in-hospital mortality of 33.9\%, compared with $13.6 \%$ for patients with shared decisions and $9.4 \%$ for patients who made their own decisions (Table 1). To investigate differences in the timing of in-hospital death, Kaplan-Meier curves were constructed showing the day of in-hospital death according to decision-maker (Figure 2A). Log rank tests showed significant differences in survival between the patient and surrogate decision groups (log-rank chi-square $=18.09, P<.001)$ but not between patient and shared decision groups (log-rank chi-square $=0.28, P=.60$ ). Controlling for demographic variables and ICU stay in Cox regression, the rate of in-hospital death was significantly higher for patients with surrogate decisions than for those with patient decisions (adjusted hazard ratio $(\mathrm{aHR})=2.61,95 \%$ confidence interval $(\mathrm{CI})=1.56-4.36)$ but was not significantly different for shared decisions and patient decisions $(\mathrm{aHR}=1.26,95 \% \mathrm{CI}=$ $0.60-2.64)$. The HR for death was also higher for patients with an ICU stay (aHR $=1.68$, $95 \% \mathrm{CI}=1.13-2.52$ ). In a separate logistic regression, patients whose DNR orders were written on Day 6 or later were twice as likely to die in the hospital $(\mathrm{OR}=2.20,95 \% \mathrm{CI}=$ $1.45-3.36)$ as those with earlier DNR orders, controlling for demographic variables and ICU stay.

To examine the proximity of the DNR order to time of death, Kaplan-Meier curves were constructed showing the time from DNR to in-hospital death as a function of the decisionmaker (Figure 2B). Log rank tests showed a significant difference between the patient and surrogate decision groups (log-rank chi-square $=30.23, P<.001$ ) but not patient and shared groups (log-rank chi-square $=1.48, P=.22$ ). Controlling for patient demographics and ICU stay, the aHR for patient death was significantly higher for surrogate than patient decisions $(3.19,95 \% \mathrm{CI}=1.94-5.26)$ but was not different for patient and shared decisions $(1.56,95 \%$ $\mathrm{CI}=0.75-3.25)$. The rate of death was also higher for patients with an ICU stay (aHR = $2.43,95 \% \mathrm{CI}=1.63-3.63)$. For patients who died, the median time from DNR order to death was 3.5 days for patients who made their own decisions, 4.0 days for shared decisions, and 1.0 day when a surrogate made the decision.

\section{DISCUSSION}

It was found that, in patients in an urban, public hospital for whom a DNR order was written during their hospital stay, surrogates made decisions without patient input more than half the time and patients made decisions independently less than one-third of the time, suggesting that, for establishing DNR status for this cohort of hospitalized older adults, surrogate decision-making is the norm rather than the exception. A lower percentage of independent patient decisions was found than in a previous study, ${ }^{8}$ which may have been because of the younger age of their population and setting in a cancer referral center rather than an acute care hospital, as in the present study. 
Research and policy addressing DNR decision-making in the hospital should account for the fact that someone other than the patient makes the majority of these decisions and should be guided by ethical theory on surrogate decision-making ${ }^{10,11,19}$ rather than models of decision-making that assume an autonomous patient. The communication and decisionmaking process need to take into account the wishes and needs of family members ${ }^{8}$ and should be guided by best practices in surrogate communication. ${ }^{20,21}$

The current study also identified important differences between patients who had a surrogate decision-maker provide consent for a DNR order and those who made the decision independently - the former were more likely to be in the ICU and more likely to die during their hospital stay. In spite of the fact that DNR status may be more appropriate as the severity of illness increases, it took longer to enter a DNR order for the more seriously ill group of patients. One other study was identified that found a similar pattern in a major cancer center. ${ }^{8}$ The findings of the current study are consistent with the literature that DNR orders for all patients are often written close to the time of death ${ }^{2,5-9}$ but also demonstrate that the time from DNR order to death is shorter for surrogate decisions than patient decisions. The shorter time frame allows less of an opportunity for family to come to terms with death before it occurs and less time to provide palliative care.

These differences between surrogate and patient decisions in the timing of DNR orders may have been because of the ethical and emotional complexities of making decisions for others. For example, surrogates often report feelings of guilt about refusing care on behalf of the patient. ${ }^{15}$ Additionally, surrogates' need for information and their preferred decision-making role may differ from patients making a DNR decision for themselves. ${ }^{22}$ There is evidence that, for surrogates, DNR decision-making is a process rather than a single event and may require more than one conversation, ${ }^{15}$ which may contribute to longer times to reach decisions.

Research has found that delays in DNR decision-making are associated with higher hospital $\operatorname{costs}^{23,24}$ and longer lengths of stay ${ }^{24,25}$ than when DNR orders are written at the time of admission. Further study is needed to determine whether supporting surrogates in their decision-making could reduce the delays in making such decisions and affect elements of patient care such as length of stay and cost.

Although differences in communication between physicians and patients and physicians and surrogates are the most likely explanation for these findings, alternative explanations exist. Some patients who had a surrogate decision-maker may have been admitted with full decision-making capacity and lost capacity late in the hospital course. For example, a person admitted for an elective cholecystectomy may become septic and delirious after the operation, requiring the family to address code status only after several days of hospitalization.

Time to DNR order was longer and time from DNR to death shorter in patients ever admitted to the ICU. This may be due to differences in goals of care; patients admitted to the ICU are more likely to have an aggressive plan of care that would include full code status. Finally, patients with late DNR orders were twice as likely to die in the hospital as those whose DNR orders were written early. The nature of later DNR discussions may be different from those that occur early. At admission, the discussion may be routine for many patients. Later, the discussion may be occurring because the patient is at imminent risk of death.

A limitation of this study is that the retrospective approach allowed examination only of cases in which the DNR order was written. There may be other patients for whom code status was discussed but who remained full code. The method of using the EMR to identify subjects did not allow these cases to be identified. Another limitation is the single hospital 
setting, which limits generalizability. Finally, information was not gathered about the extent or quality of communication regarding the DNR decision, so whether there are differences in the communication or decision-making with patients compared to surrogates was not examined.

In conclusion, surrogates were involved in the majority of DNR decisions for hospitalized older adults in an urban public hospital. The longer time required for surrogate decisions may be because of the ethical and emotional complexities of making decisions for others. DNR decisions made so close to a patient's death may reduce the time available for quality end of life care. Future research should identify the communicative and ethical factors that require additional time for surrogate decision-making and should develop and evaluate interventions to optimize the decision-making process so as to avoid any unnecessary delays in the writing of appropriate DNR orders for older adults who require a surrogate decisionmaker.

\section{Acknowledgments}

The authors would like to acknowledge Timothy E. Stump for biostatistics assistance, Evgenia Teal for assistance with data management, Bridget Callahan for assistance with literature review, and Cynthia Hamilton for assistance with manuscript preparation.

Sponsor's Role: These funders had no role in the design, methods, subject recruitment, data collections, analysis, or preparation of the paper.

\section{References}

1. Auerbach AD, Katz R, Pantilat SZ, et al. Factors associated with discussion of care plans and code status at the time of hospital admission: Results from the Multicenter Hospitalist Study. J Hosp Med. 2008; 3:437-445. [PubMed: 19084893]

2. Morrell ED, Brown BP, Qi R, et al. The do-not-resuscitate order: Associations with advance directives, physician specialty and documentation of discussion 15 years after the Patient SelfDetermination Act. J Med Ethics. 2008; 34:642-647. [PubMed: 18757631]

3. Bedell SE, Delbanco TL. Choices about cardiopulmonary resuscitation in the hospital. When do physicians talk with patients? N Engl J Med. 1984; 310:1089-1093. [PubMed: 6708990]

4. Tulsky JA, Chesney MA, Lo B. How do medical residents discuss resuscitation with patients? J Gen Intern Med. 1995; 10:436-442. [PubMed: 7472700]

5. Jonsson PV, McNamee M, Campion EW. The 'Do not resuscitate' order. A profile of its changing use. Arch Intern Med. 1988; 148:2373-2375. [PubMed: 3190371]

6. Gleeson K, Wise S. The do-not-resuscitate order. Still too little too late. Arch Intern Med. 1990; 150:1057-1060. [PubMed: 2331186]

7. Maksoud A, Jahnigen DW, Skibinski CI. Do not resuscitate orders and the cost of death. Arch Intern Med. 1993; 153:1249-1253. [PubMed: 8494477]

8. Levin TT, Li Y, Weiner JS, et al. How do-not-resuscitate orders are utilized in cancer patients: Timing relative to death and communication-training implications. Palliat Support Care. 2008; 6:341-348. [PubMed: 19006588]

9. Zimmerman JE, Knaus WA, Sharpe SM, et al. The use and implications of do not resuscitate orders in intensive care units. JAMA. 1986; 255:351-356. [PubMed: 3941514]

10. Torke AM, Alexander GC, Lantos J, et al. The physician-surrogate relationship. Arch Intern Med. 2007; 167:1117-1121. [PubMed: 17563018]

11. Dubler NN. The doctor-proxy relationship: The neglected connection. Kennedy Inst Ethics J. 1995; 5:289-306. [PubMed: 10153758]

12. Baker R, Wu AW, Teno JM, et al. Family satisfaction with end-of-life care in seriously ill hospitalized adults. J Am Geriatr Soc. 2000; 48:S61-S69. [PubMed: 10809458] 
13. Torke AM, Siegler M, Abalos A, et al. Physicians' experiences with surrogate decision making for hospitalized adults. J Gen Intern Med. 2009; 24:1023-1028. [PubMed: 19633896]

14. Azoulay E, Pochard F, Kentish-Barnes N, et al. Risk of post-traumatic stress symptoms in family members of intensive care unit patients. Am J Respir Crit Care Med. 2005; 171:987-994. [PubMed: 15665319]

15. Handy CM, Sulmasy DP, Merkel CK, et al. The surrogate's experience in authorizing a do not resuscitate order. Palliat Support Care. 2008; 6:13-19. [PubMed: 18282340]

16. Raymont V, Bingley W, Buchanan A, et al. Prevalence of mental incapacity in medical inpatients and associated risk factors: Cross-sectional study. Lancet. 2004; 364:1421-1427. [PubMed: 15488217]

17. Stolman CJ, Gregory JJ, Dunn D, et al. Evaluation of the do not resuscitate orders at a community hospital. Arch Intern Med. 1989; 149:1851-1856. [PubMed: 2764656]

18. Atkins DC. Rethinking how family researchers model infrequent outcomes: A tutorial on count regression and zero-inflated models. J Fam Psychol. 2007; 21:729-735.

19. Buchanan, B. Deciding for Others: The Ethics of Surrogate Decision Making. Cambridge, UK: Cambridge University Press; 1990.

20. Karlawish JH, Quill T, Meier DE. A consensus-based approach to providing palliative care to patients who lack decision-making capacity. ACP-ASIM End-of-Life Care Consensus Panel. American College of Physicians-American Society of Internal Medicine. Ann Intern Med. 1999; 130:835-840. [PubMed: 10366374]

21. White DB, Malvar G, Karr J, et al. Expanding the paradigm of the physician's role in surrogate decision-making: An empirically derived framework. Crit Care Med. 2010; 38:743-750. [PubMed: 20029347]

22. Heyland DK, Frank C, Groll D, et al. Understanding cardiopulmonary resuscitation decision making: Perspectives of seriously ill hospitalized patients and family members. Chest. 2006; 130:419-428. [PubMed: 16899840]

23. De Jonge KE, Sulmasy DP, Gold KG, et al. The timing of do-not-resuscitate orders and hospital costs. J Gen Intern Med. 1999; 14:190-192. [PubMed: 10203626]

24. Celso BG, Meenrajan S. The triad that matters: Palliative medicine, code status, and health care costs. Am J Hosp Palliat Care. 2010; 27:398-401. [PubMed: 20332499]

25. Hakim RB, Teno JM, Harrell FE, et al. Factors associated with do-not-resuscitate orders: Patients' preferences, prognoses, and physicians' judgments. SUPPORT Investigators. Study to Understand Prognoses and Preferences for Outcomes and Risks of Treatment. Ann Intern Med. 1996; 125:284-293. [PubMed: 8678391] 


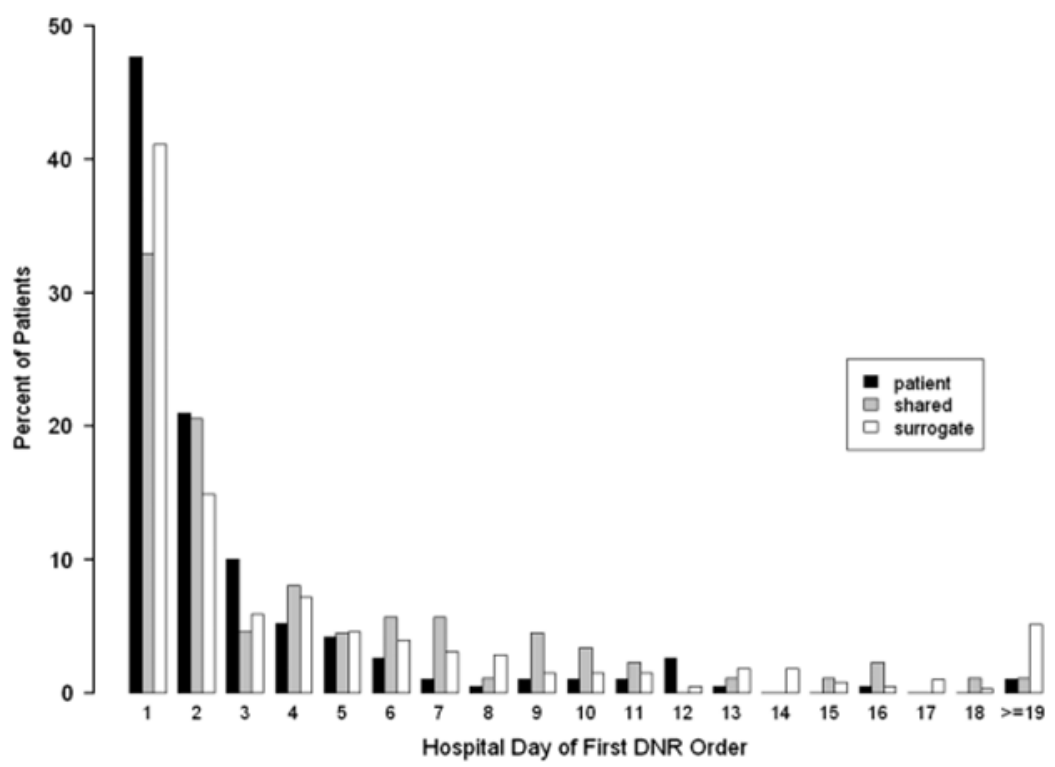

Figure 1.

Frequency distribution of number of days to first do-not-resuscitate (DNR) order according to decision group. 

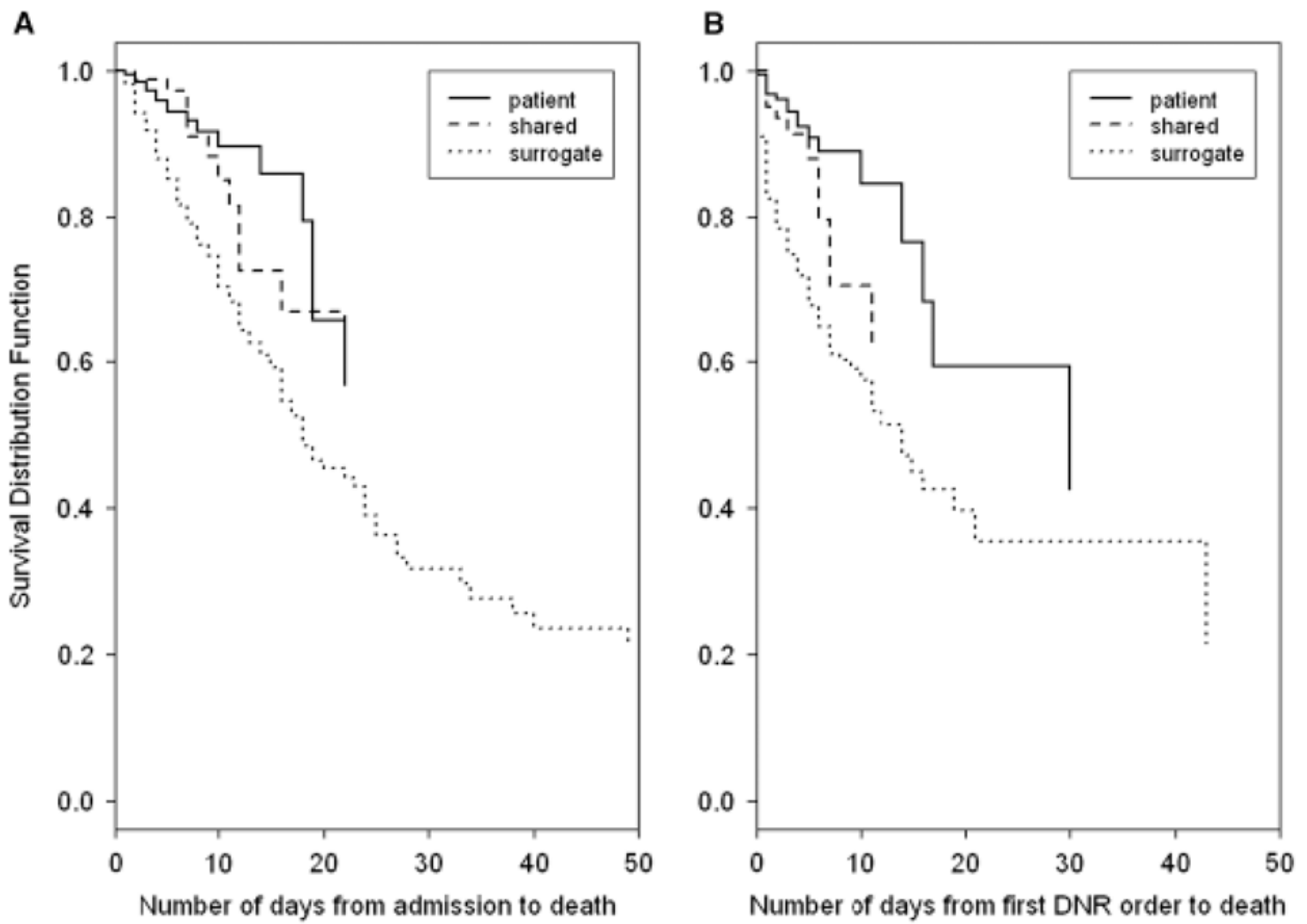

Figure 2.

Kaplan-Meier survival plots for days from admission to death (A) and days from first donot-resuscitate (DNR) order to death (B). 


\section{Table 1}

Participant Characteristics, Hospital Course, and Outcomes for All Participants and According to DecisionMaker for Do-Not-Resuscitate Order

\begin{tabular}{|c|c|c|c|c|}
\hline Variable & All Decisions & Patient Decisions & Shared Decisions & Surrogate Decisions \\
\hline Observations, n (\%) & 668 & 191 (28.6) & $88(13.2)$ & $389(58.2)$ \\
\hline \multicolumn{5}{|l|}{ Age, $\%$} \\
\hline $65-74$ & 35.0 & 41.4 & 36.4 & 31.6 \\
\hline $75-84$ & 42.1 & 42.4 & 40.9 & 42.2 \\
\hline 285 & 22.9 & 16.2 & 22.7 & 26.2 \\
\hline \multicolumn{5}{|l|}{ Sex, n (\%) } \\
\hline Male & $239(35.8)$ & $70(36.7)$ & $24(27.3)$ & $145(37.3)$ \\
\hline Female & $429(64.2)$ & $121(63.4)$ & $64(72.7)$ & $244(62.7)$ \\
\hline \multicolumn{5}{|l|}{ Race, n (\%) } \\
\hline African American & $335(50.2)$ & $89(46.6)$ & $41(46.6)$ & $205(52.7)$ \\
\hline White & $301(45.1)$ & $95(49.7)$ & $40(45.5)$ & $166(42.7)$ \\
\hline Hispanic, Latino, or Asian & $32(4.8)$ & $7(3.7)$ & $7(8.0)$ & $18(4.6)$ \\
\hline Any Medicaid, n (\%) & $468(70.1)$ & $136(71.2)$ & $61(69.3)$ & $271(69.7)$ \\
\hline Intensive care unit stay, $\mathrm{n}(\%)$ & $354(53.0)$ & $76(39.8)$ & $36(40.9)$ & $242(62.2)^{*}$ \\
\hline Length of stay, mean $\pm \mathrm{SD}$ & $10.1 \pm 21.1$ & $7.8 \pm 9.1$ & $8.8 \pm 7.3$ & $11.6 \pm 26.6^{\dagger}$ \\
\hline Hospital charges (in $\$ 1,000$ s) mean \pm SD & $27.8 \pm 46.0$ & $17.3 \pm 28.6$ & $22.0 \pm 26.9^{*}$ & $34.3 \pm 54.5^{*}$ \\
\hline In-hospital mortality, n (\%) & $162(24.3)$ & $18(9.4)$ & $12(13.6)$ & $132(33.9)^{*}$ \\
\hline 30-day mortality, n (\%) & $298(44.6)$ & $59(30.9)$ & $35(39.8)$ & $204(52.4)^{*}$ \\
\hline \multicolumn{5}{|l|}{$P<* .001$} \\
\hline
\end{tabular}

